\title{
MIGRATION AND PROLIFERATION ANALYSIS FOR BLADDER CANCER CELLS
}

\author{
Asaad F. Said( ${ }^{(1)}$, Lina J. Karam ${ }^{(1)}$, Zoe Lacroix ${ }^{(1)}$, Michael E. Berens ${ }^{(2)}$, Rosemary A. Renaut ${ }^{(3)}$ \\ (1) Department of Electrical Engineering, Arizona State University, Tempe, AZ 85287-5706 \\ (2) TGen, Translational Genomics Research Institute, Phoenix, AZ 85004 \\ (3) Department of Mathematics and Statistics, Arizona State University, Tempe, AZ 85287-1804 \\ \{asaad.said, karam, zoe.lacroix\}@asu.edu,mberens@tgen.org, renaut@asu.edu
}

\begin{abstract}
This paper presents a cell evolution analysis (CEA) scheme for bladder cancer cells. The proposed scheme consists of a cell migration analysis component for computing the overall migration rate of the cell cluster, and a cell proliferation analysis component based on counting the individually segmented cells within the cell cluster at different time points. The proposed CEA scheme performs well for images with poor contrast and high cell concentrations, even when the cells are overlapping and small. Results are presented to illustrate the performance of the proposed scheme.
\end{abstract}

\section{INTRODUCTION}

Studying the migration and proliferation behavior of cells contributes to the understanding of biological processes and to disease pathologies such as cancer, angiogenesis, vascular stenosis and arthritis. To this end, cellular imaging coupled with image processing and segmentation techniques, has been used to provide an automatic and improved analysis of the cellular behavior.

Cell segmentation is one of the many challenging tasks in cellular image processing. Several methods have been proposed and developed for cell segmentation and tracking [1-7]. In general, these methods work for specific cell types and under specific constraints. In [1], a gradient-based levelset method is used for cluster segmentation of neural stem cells. But the method of [1] is not robust in the presence of noise. Moreover, this method is not fully automatic as it requires knowledge of the cell cluster location relative to the initial boundary of the evolving level-set function. In [2], a cell tracking scheme is presented, for in vitro phase-contrast video microscopy, using a combination of mean-shift processes. However, in the presented method [2], the user needs to manually select the locations of the cells in the first or the last frame of the video sequence. In addition, the original frames need to be pre-processed by performing contrast enhancement and illumination correction. In [3], a cell cluster segmentation algorithm is presented based on global and local thresholding for In-SITU microscopic images. This method requires noise-free images, nonoverlapping cells, and a high-contrast between cells and background. Also, many parameters need to be adjusted to compute the local threshold. In [4], the level-set method of [8] is used, with one level-set function, for the segmentation and tracking of multiple motile epithelial cells during wound healing. In [5], a topology-constrained level-set method is presented to prevent the merging of touching and partially overlapping cells. Level-set methods were also used for cell segmentation and tracking in [6]. In [7], a probabilistic model was proposed for the segmentation of hematopoietic stem cells; the proposed model is based on identifying the most probable cell locations in the image on the basis of cell brightness and morphology. The method [7] is sensitive to cell overlap, cell shape, and the used threshold. Moreover, the methods of [5-7] require noise-free images with a good contrast between the cells and background.

In this paper, a Cell Evolution Analysis (CEA) scheme is presented for the automatic segmentation and analysis of the overall migration rate and proliferation behavior of bladder cancer cells. In contrast to existing cell segmentation and analysis methods, the proposed method performs well in the presence of noise; it also performs well for images with poor contrast and high cell concentrations, even when the cells are overlapping and small. The presented method was successfully applied on noisy and poor-contrast images of 34 different bladder cancer cell lines interacting on different matrix substrates at two time points (16 and 40 hours). The cells in these images are also very small in size and heavily concentrated within a portion of the image. A sample image is shown in Fig. 3(a). For visual clarity, an enhanced version of this image is also shown in Fig. 3(b); however, only the non-enhanced original images are directly processed by our proposed CEA scheme.

This paper is organized as follows. The proposed Cell Evolution Analysis (CEA) scheme is described in Section 2. The proposed CEA scheme includes methods for cell cluster segmentation and cell counting in order to determine, respectively, the overall migration rate of the cell cluster and 
the cell proliferation behavior. Details about the proposed cell cluster segmentation and cell counting algorithms are given in Sections 3 and 4, respectively. Results are presented in Section 5, and a conclusion is given in Section 6.

\section{PROPOSED CELL EVOLUTION ANALYSIS (CEA) SCHEME}

The proposed cell evolution analysis (CEA) scheme consists of the following two main parts:

1- Cell migration analysis- achieved by determining and segmenting the region where the cells are concentrated, and then tracking the evolution of the contour of that region over time. We refer to this cell cluster region as the region-of-interest (ROI).

2- Cell proliferation analysis- achieved by segmenting and counting the individual cells in the ROI at different time points.

As shown in Figs. 2(a) and 2(b), the considered bladder cancer cell images consist, each, of three different regions: 1) the ROI, 2) the region between the ROI and the outer circle (bright area), and 3 ) the region outside the outer circle (dark area). We need to find an automatic segmentation method to distinguish between these three regions. For the level-set based segmentation methods, two level-set functions are needed for the considered images. The levelset method of [4] is, consequently, not applicable. Alternatively,, when the scheme of Chen \& Vese [8], with two level set functions, is applied directly to these images, it only succeeds in capturing the contour of the outer circle, but fails to capture the contour of the ROI. This occurs becausethee considered ROI is not a piecewise constant area and contains many gaps between the cells.

A block diagram of the proposed cell evolution analysis (CEA) scheme is shown in Fig. 1. The proposed CEA scheme proceeds as follows. For each image cell, a cell merging operation is first performed to fill the gaps between cells; this is done using a closing operation with a circular structural element. Also, if noise is present, spatial smoothing is performed using a simple averaging filter. This is followed by a level-set-based segmentation technique which makes use of two level-set functions for extracting the ROI in addition to the outer circle contour. The resulting segmented image can include small regions due to artifacts in the original image; these can be easily discarded due to their small size relative to the ROI. The overall migration rate of the cells (Cell migration analysis block in Fig. 1) is performed by tracking the evolution of the ROI size at different times. The size and shape of the ROI in one image changes with time due to the duplication and migration of cells. From the segmented ROIs at two successive times $t_{a}$ and $t_{b}$, where $t_{b}>t_{a}$, the cell cluster migration rate can be computed as $M R=\left(A\left(t_{b}\right)-A\left(t_{a}\right)\right) /\left(t_{b}-t_{a}\right)$, where $A\left(t_{i}\right)$ is the area inside the ROI at time $t_{i}$. The cell proliferation behavior (Cell proliferation analysis block in Fig. 1) is analyzed through a cell counting procedure that is applied to the extracted ROIs at different time points. Details about the level-set-based ROI segmentation and the cell counting procedures are given in Sections 3 and 4, respectively.

\section{LEVEL-SET-BASED ROI SEGMENTATION}

Level-set methods provide powerful mathematical tools to solve many geometrical problems such as topological changes, and tracking the contour of objects. Two level-set based methods, the weighted level-set method and the twostep level-set method, are proposed for the ROI segmentation. Both methods gave the same segmentation results on the considered images; but the two-step level-set segmentation method converges more quickly.

\subsection{Weighted level-set method}

This method is a modified version of the method with twolevel functions presented in [8]. In this modified version, the following weighted energy function is minimized:

$$
\begin{aligned}
& F_{4}\left(c, \phi_{1}, \phi_{2}\right)=\lambda_{1} \Omega_{0}\left|u_{0}-c_{11}\right|^{2} H\left(\phi_{1}\right) H\left(\phi_{2}\right) d x d y \\
& +\lambda_{\Omega}\left|u_{0}-c_{10}\right|^{2} H\left(\phi_{1}\right)\left(1-H\left(\phi_{2}\right)\right) d x d y \\
& +\lambda_{3}\left|u_{0}-c_{01}\right|^{2} H\left(\phi_{2}\right)\left(1-H\left(\phi_{1}\right)\right) d x d y \\
& +\lambda_{4}\left|u_{0}-c_{00}\right|^{2}\left(1-H\left(\phi_{1}\right)\right)\left(1-H\left(\phi_{2}\right)\right) d x d y \\
& +v_{1}\left|H\left(\phi_{1}\right)\right|+v_{2}\left|H\left(\phi_{2}\right)\right|
\end{aligned}
$$

where $\lambda^{\prime} s$ and $v^{\prime} s$ are constants, $u_{0}$ is the considered image, and $c_{11}, c_{10}, c_{01}, c_{00}$ are constants corresponding to the mean of the image $u_{0}$ in the regions $\left\{\phi_{1}>0, \phi_{2}>0\right\}$, $\left\{\phi_{1}>0, \phi_{2}<0\right\},\left\{\phi_{1}<0, \phi_{2}>0\right\}$, and $\left\{\phi_{1}<0, \phi_{2}<0\right\}$, respectively [8]. $\phi_{1}$ and $\phi_{2}$ are the two level set functions and these evolve based on minimizing the energy in (1) to segment the image $u_{0}$. The method of [8] (which corresponds to setting the weights $\lambda_{i}=1, i=1, \ldots, 4$ in (1)) fails to segment the considered bladder cancer cell images even after performing the cell merging operation (Section 2). Instead, the ROI is partitioned into different regions by $\phi_{1} \& \phi_{2}$. This is due to the fact that the ROI has different means instead of one constant mean, even after applying the cell merging operation. To overcome this problem, the proposed weighted level-set segmentation method forces only one level set function to evolve inside the ROI by setting the weights in (1) to $\lambda_{1}=\lambda_{2}=\lambda_{a} \& \lambda_{3}=\lambda_{4}=1$. Using these weight values, it can be easily shown that the evolutions of the level set functions $\phi_{1}, \phi_{2}$ are given by: 


$$
\begin{aligned}
& \frac{\phi_{1}}{t}=\delta_{\varepsilon}\left(\phi_{1}\right) \quad v_{1} \cdot \frac{\phi_{1}}{\left|\phi_{1}\right|}-\left(\lambda_{a}\left(u_{0}-c_{11}\right)^{2}-\left(u_{0}-c_{01}\right)^{2}\right) H\left(\phi_{2}\right) \\
& -\left(\lambda_{a}\left(u_{0}-c_{10}\right)^{2}-\left(u_{0}-c_{00}\right)^{2}\right)\left(1-H\left(\phi_{2}\right)\right) \\
& \frac{\phi_{2}}{t}=\delta_{\varepsilon}\left(\phi_{2}\right) \quad v_{2} \cdot \frac{\phi_{2}}{\left|\phi_{2}\right|}-\lambda_{a}\left(\left(u_{0}-c_{11}\right)^{2}-\left(u_{0}-c_{10}\right)^{2}\right) H\left(\phi_{1}\right) \\
& -\left(\left(u_{0}-c_{01}\right)^{2}-\left(u_{0}-c_{00}\right)^{2}\right)\left(1-H\left(\phi_{1}\right)\right)
\end{aligned}
$$

In (2), when choosing $\lambda_{a}>1$, more weight is given for the region inside $\phi_{1}\left(\phi_{1}>0\right)$ to evolve inside $\varphi_{2}\left(\phi_{2}>0\right)$. In this way, after few iterations, $\varphi_{1}$ starts to evolve inside $\varphi_{2}$ to capture the ROI, while $\varphi_{2}$ evolves to capture the outercircle. We applied this method to the experimental cell bladder cancer images and obtained good ROI segmentation results. The disadvantages of this method are: the computation time, the selection of the parameter $\lambda_{a}$, and the coupling between $\phi_{1} \& \phi_{2}$ [9].

\subsection{Two-step level-set method}

The level-set method of [9] is adopted and applied to the considered bladder cancer cell images after the cell merging operation, as discussed in Section 2. This method applies $\phi_{1}$ and $\phi_{2}$, consecutively, rather than simultaneously. This process ensures that $\phi_{1}$ and $\phi_{2}$ are completely decoupled. This method proceeds in two steps as follows:

Step 1 - Evolve one level-set function $\phi_{1}$ to segment the outer-circle using:

$$
\frac{\phi_{1}}{t}=\delta_{\varepsilon}\left(\phi_{1}\right) \quad v_{1} \cdot \frac{\phi_{1}}{\left|\phi_{1}\right|}-\left(u_{0}-c_{1}^{I}\right)^{2}+\left(u_{0}-c_{2}^{I}\right)^{2}
$$

where $c_{1}^{I}$ and $c_{2}^{I}$ are the averages of $u_{0}$ inside and outside $\phi_{1}$, respectively. The outer-circle is the region inside $\phi_{1}\left(\phi_{1}>0\right)$.

Step 2 - Evolve level-set function $\phi_{2}$ inside $\phi_{1}\left(\phi_{1}>0\right)$ to segment the ROI using:

$$
\frac{\phi_{2}}{t}=\delta_{\varepsilon}\left(\phi_{2}\right) \quad v_{2} \cdot \frac{\phi_{2}}{\left|\phi_{2}\right|}-\lambda_{1}\left(u_{0}-c_{1}^{I I}\right)^{2}+\lambda_{2}\left(u_{0}-c_{2}^{I I}\right)^{2}
$$

where $c_{1}^{I I}$ and $c_{2}^{I I}$ are the averages of $u_{0}$ inside and outside $\phi_{2}$, respectively, when $\phi_{1}>0$. In (4), the values of $\lambda_{1}$ and $\lambda_{2}$ affect the evolution rate of $\phi_{2}$ [9].

The advantages of this two-step level-set method, as compared to the weighted level-set method of Section 3.1, include a faster speed of convergence, lower computational complexity, and insensitivity to the initial conditions. For each step, the level-set function evolution is stopped when the absolute difference between the current and previous level-set function is less than a certain threshold.

\section{CELL SEGMENTATION \& COUNTING}

Cell counting is used in cell tracking and cell proliferation analysis. In the considered images, the cell population inside the ROI is in the range of $600-8000$ cells/image. Due to a large number of images and cells, manual counting of cells by humans has proven to be impractical, non-reliable, and exhaustive. So, an automatic reliable counting scheme is essential for speed and accuracy. Several automatic or semi-

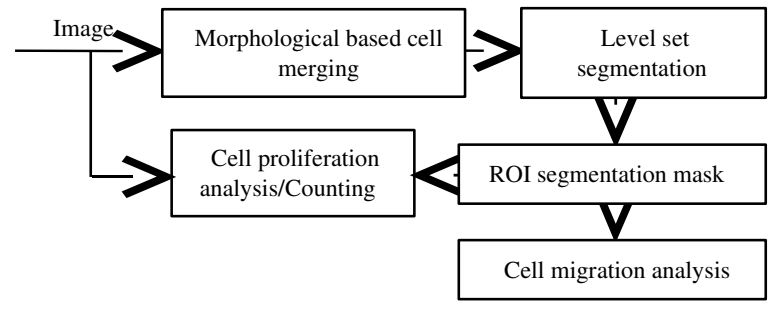

Fig.1. Block diagram of the proposed CEA scheme.

automatic methods have been proposed to segment the boundaries of the cells, and then count them. In [10], an $a$ trous wavelet transform is used to detect spots in the presence of noise. But this method works under certain assumptions such as a smooth background, and a good contrast between cells and background, and disconnected cells. In [11], a comparison presented for 7 thresholding methods for breast tumor cell segmentation, found that . Otsu's method performed best. Otsu's method, however, does not give satisfactory results if the image contrast is low. Most of the existing methods fail to segment or count the cells if these are connected. In [5], the images have a high contrast between the cells and background, and cell populations are in the range $350-750$ cells/image, which is significantly lower than the cell density in the bladder cancer cell images considered in this work. The main challenges with the given bladder cancer cell images are: heavily populated (high cell density), tiny cells, touching and overlapping cells, presence of noise in the images, and very low contrast as the intensity values of cell boundaries and background are very close. From Fig. 3(e), it can be noticed that the centroid (nucleus) of the cell has a lower intensity than the cell boundary (halo). Consequently, we use a strategy in which we count the centroids of the cells instead of counting based on the cell boundary [2]; in this way, we can also separate connected and overlapping cells by their centroids as long as the centroids are separated.

The proposed cell segmentation and counting procedure can be summarized as follows: 
1- Using the segmentation masks of the outer-circle and of the ROI, which are obtained as described in Sections 2 and 3, the histogram, mean, and variance of the background and of the ROI are computed. The background here is the region between the ROI and the outer-circle.

2- From the mean and variance of the background and the ROI, a threshold is computed and used to classify the ROI into significant (cells' centroids) and nonsignificant regions.

3- The resulting connected significant regions are counted using a labeling procedure.

The histogram distributions of the background (dashed curve) and ROI (solid curve) are shown in Fig. 2 for one of the considered images. It can be seen that the background and the ROI are close in mean. However, the background has a small variance as compared to the variance of the ROI. The increase of the variance, inside the ROI, is due to the large population of cells and the intensity variations between the centroid and the surface of the cell. Since the nonsignificant regions in the ROI (ROI pixels that do not correspond to a cell's centroid) have intensities similar to

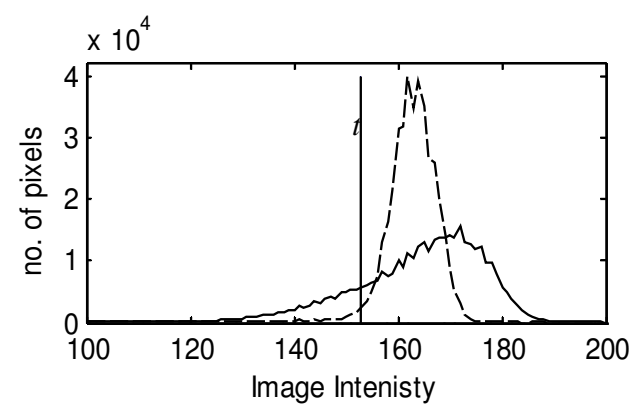

Fig. 2. Background (dashed) and ROI (solid)

histograms.

the background region (region between ROI and outer circle) and since the background has a relatively low variance, a threshold can be computed based on the mean and standard deviation of the background to segment the cells' centroids (significant ROI regions) from the nonsignificant ROI background-like regions [2]. The classification threshold is computed as $t=\mu_{b g}-\delta_{b s}$, where $\mu_{b g}$ and $\delta_{b g}$ represent the mean and standard deviation of the background, respectively. A pixel in the ROI is classified as significant (as belonging to a cell's centroid) if its intensity is less than the threshold $t$; otherwise, it is classified as nonsignificant. A labeling process is then applied to count the obtained cell centroids.

\section{EXPERMENTAL RESULTS}

The proposed CEA scheme has been successfully tested using 120 bladder cancer cell images of size 1030x1300 (60 different images at two different time points 16 and 40 hours). Fig. 3 shows the results obtained using one of the images at 40 hours. The original input image is shown in Fig. 3(a). It can be seen that it is hard to clearly distinguish the cells from the background due to their small size and the low contrast. For illustration and visual clarity, an enhanced version of Fig. 3(a) is shown in Fig. 3(b), although the proposed scheme is applied directly on the original lowcontrast and non-enhanced image (Fig. 3(a)). The ROI segmentation result is shown in Fig. 3(c) (white contour for outer-circle, and the black contour for the ROI). The number of iterations needed to segment the outer-circle is 3-4 iterations by using (3), and 4-6 iterations to segment the ROI by using (4). In comparison, the weighted level-set method requires at least 20 iterations to segment the outer-circle and the ROI by using (2). The resulting segmented ROI and an enlarged portion of the ROI are shown in Figs. 3(d) and 3(e), respectively. From Fig. 3(e), it can be seen that the ROI contains touching and overlapping cells with a low contrast between the cells and their surround. Despite the low

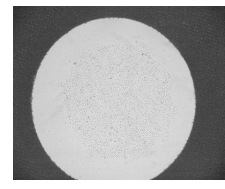

(a) Original image

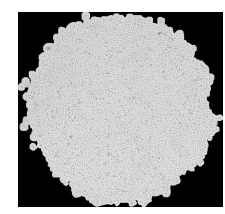

(d) Extracted ROI

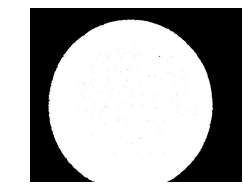

(b) Enhanced version of (a)

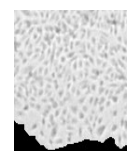

(e) Enlarged part of ROI

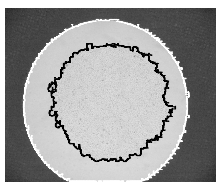

(c) Level-set segmentation

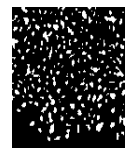

(f) Cell Segmentation of(e)
Fig. 3. ROI and cell segmentation results using proposed scheme.

contrast and cell overlap, the proposed automatic thresholding procedure (Section 4) is able to successfully separate the cells for counting by segmenting their centroids as shown in Fig. 3(f). The obtained counted number of cells inside the ROI is 7,073 for the considered image. The computed area of the extracted ROI is 378,375 pixel $^{2}$.

\section{CONCLUSION}

This paper presents a scheme for the analysis of the migration and proliferation behavior of bladder cancer cells. The proposed scheme was successfully tested on bladder cancer cell images with low contrast, cell overlap, and noise. 
Future work will include testing and extending this scheme to other cell types in addition to tracking individual cells.

\section{REFERENCES}

[1] N.N. Kachouie and P. Fieguth, "A Narrow-Band Level-Set Method with Dynamic Velocity for Neural Stem Cell Cluster Segmentation" Image Analysis and Recognition, $2^{\text {nd }}$ Int. Conf., ICIAR 2005, Toronto, Canada, September 28-30, 2005

[2] O. Debeir, P.V. Ham, R. Kiss, and C. Decaestecker, "Tracking of Migrating Cells Under Phase-Contrast Video Microscopy With Combined Mean-Shift Processes", IEEE

Trans. on Medical Imaging,Vo.24, No.6, pp. 697-711, June 2005.

[3] E. Espinoza, G. Martinez, J.-G. Frerichs, and T. Scheper, "Cell Cluster Segmentation Based on Global and Local Thresholding for In-Situ Microscopy", IEEE Int. Symp. on Biomedical Imaging, ISBI, Virginia, USA, April, 2006.

[4] F. Bunyak, K. Palaniappan, S.K. Nath, T. Baskin, and G. Dong, "Quantitative Cell Motility for In Vitro Wound Healing Using Level Set-Based Active Contour Tracking", IEEE Int. Symp. on Biomedical Imaging, ISBI, Virginia, USA, April 6-9, 2006.

[5] K. Li, E.D. Miller, L.E. Weiss, P.G. Campbell, and T. Kanade, "Online Tracking of Migrating and Proliferating Cells Imaged with Phase-Contrast Microscopy",IEEE Computer Society Workshop on Mathematical Methods in Biomedical Image Analysis, New York City, NY, USA, June 2006.

[6] F. Yang, M.A. Mackey, F. Ianzini, G. Gallard, and M. Sonka, "Cell Segmentation, Tracking, and Mitosis Detection using Temporal Context", Proc. of Int. Conf. Medical Image Computing and Computer-Assisted Intervention, MICCAI 2005, Palm Springs, California, USA, Oct. 2005.

[7] N.N. Kachouie, L.J Lee, and P. Fieguth, "A probabilistic living cell segmentation model", IEEE Inter. Conf. on Image Proc., ICIP 2005,Vol.1, September 2005.

[8] L. Vese and T. F. Chan, "A multiphase level set framework for image segmentation using the Mumford and Shah model," Int. J. Computer Vision, vol. 50, no. 3, pp. 271-293, 2002.

[9] S. Gao and T.D. Bui, "Image Segmentation and Selective Smoothing by Using Mumford-Shah Model", IEEE Trans. Image Process., vol.14, no.10, pp.1537-1549, Oct. 2005.

[10] J.C. Olivo-Marin, "Extraction of spots in biological images using multiscale products", Pattern Recognition, Vol.35, no.9, September 2002.

[11] H.J. Jeong, T.Y. Kim, H.G. Hwang, H.J. Choi, H.S. Park; and H.K. Choi, "Comparison of thresholding methods for breast tumor cell segmentation", $7^{\text {th }}$ Int. Workshop on Enterprise networking and Computing in Healthcare Industry, Busan, Korea, June 2005. 